\title{
Neoadjuvant pyrotinib plus nab-paclitaxel, doxorubicin, and cyclophosphamide for HER2-positive locally advanced breast cancer: a retrospective case-series study
}

\author{
De-Shun Yao ${ }^{1}$, Wei Wang ${ }^{2}$, Jin-Yi Chang ${ }^{2}$, Yang Zhang ${ }^{1}$, Hui-Wen Zhang ${ }^{3}$, Jin-Xia Xu ${ }^{4}$, Hai-Feng Cai ${ }^{1}$ \\ ${ }^{1}$ Second Department of Breast Surgery, Tangshan People's Hospital, North China University of Science and Technology, Tangshan, China; ${ }^{2}$ School \\ of Clinical Medicine, North China University of Science and Technology, Tangshan, China; ${ }^{3}$ College of Nursing and Rehabilitation, North \\ China University of Science and Technology, Tangshan, China; ${ }^{4}$ Nuclear Medicine Clinical Laboratory, Tangshan People's Hospital, North China \\ University of Science and Technology, Tangshan, China \\ Contributions: (I) Conception and design: HF Cai; (II) Administrative support: HF Cai; (III) Provision of study materials or patients: DS Yao, W \\ Wang, HW Zhang, JX Xu; (IV) Collection and assembly of data: DS Yao, JY Chang; (V) Data analysis and interpretation: Y Zhang, DS Yao; (VI) \\ Manuscript writing: All authors; (VII) Final approval of manuscript: All authors. \\ Correspondence to: Hai-Feng Cai. Second Department of Breast Surgery, Tangshan People's Hospital, North China University of Science and \\ Technology, Shengli Road 65, Tangshan 063000, China. Email: 13303050005@163.com.
}

Backgroundk The anti-tumor activity of pyrotinib has been confirmed in human epidermal growth factor receptor 2 (HER2)-positive metastatic breast cancer. This study investigated the effect of pyrotinib plus nabpaclitaxel, doxorubicin, and cyclophosphamide as neoadjuvant therapy in patients with HER2-positive locally advanced breast cancer.

Methods: In this single-center retrospective study, female patients with HER2-positive locally advanced breast cancer received pyrotinib $320 \mathrm{mg}$ orally once a day and the TAC regimen (nab-paclitaxel $260 \mathrm{mg} / \mathrm{m}^{2}$, liposomal doxorubicin $20 \mathrm{mg} / \mathrm{m}^{2}$, and cyclophosphamide $600 \mathrm{mg} / \mathrm{m}^{2}$ ) on day 1 of each 21-day cycle. Surgery was performed after 4-6 cycles of neoadjuvant therapy. The outcomes included total pathological complete response (tpCR, ypT0/Tis ypN0) rate, objective response rate (ORR) after neoadjuvant therapy, progressionfree survival, overall survival, and the incidence of adverse events (AEs).

Results: Between March 2019 and January 2020, a total of 22 patients were included. The median age was 48 years (range, 32-60). The ORR was $100 \%$ after the completion of neoadjuvant therapy. Ten (45.5\%) patients achieved tpCR, including four of ten $(40.0 \%)$ patients with positive hormone receptor, and six of 12 $(50.0 \%)$ patients with negative hormone receptor. As at December 2020, no disease recurrence, progression, or death occurred. All patients suffered AEs after neoadjuvant therapy, most of which were grade $1-2$. Grade $\geq 3$ AEs included diarrhea [4 (18.2\%)], rash [2 (9.1\%)], and hand-foot syndrome [1 (4.5\%)].

Conclusions: Neoadjuvant pyrotinib combined with the TAC regimen showed promising clinical benefit in patients with HER2-positive locally advanced breast cancer, with an acceptable safety profile.

Keywords: Pyrotinib; chemotherapy; neoadjuvant therapy; human epidermal growth factor receptor 2; breast cancer

Submitted Oct 29, 2021. Accepted for publication Dec 16, 2021.

doi: $10.21037 /$ gs-21-770

View this article at: https://dx.doi.org/10.21037/gs-21-770 


\section{Introduction}

According to 2020 global estimates of the incidence and mortality for 36 cancers in 185 countries, female breast cancer has surpassed lung cancer as the leading cause of newly diagnosed cancer worldwide (1). The latest statistical report in China showed that the incidence of female breast cancer was 304,000 in 2015 (2). Human epidermal growth factor receptor 2 (HER2)-positive breast cancer is an aggressive subtype, with higher risks of recurrence and metastasis, and worse prognosis $(3,4)$. The use of antiHER2 monoclonal antibody as (neo)adjuvant therapy has improved survival in patients with HER2-positive early or locally advanced breast cancer (5-10).

The current recommended neoadjuvant regimens in guidelines are chemotherapy combined with dual-targeted therapy (trastuzumab and pertuzumab) or mono-targeted therapy (trastuzumab) $(11,12)$. However, some HER2positive breast cancer patients, especially those with cardiovascular comorbidities, cannot use the large-molecule monoclonal antibody and anthracycline due to cardiac safety concerns. At the same time, new agents are emerging and their efficacy had been confirmed in metastatic and/ or (neo)adjuvant settings. The phase 3 NeoALTTO trial demonstrated that dual inhibition of HER2 with lapatinib plus trastuzumab brought a significantly higher total pathological complete response (tpCR) rate than trastuzumab alone ( $46.8 \%$ vs. $27.6 \%)$, combined with paclitaxel in the neoadjuvant setting for HER2-positive early breast cancer (13). In the neoadjuvant phase 2 I-SPY 2 trial, the addition of neratinib to paclitaxel resulted in a higher tpCR rate than trastuzumab plus paclitaxel $(39 \%$ vs. $23 \%$ ) in patients with HER2-positive early breast cancer (14). The emergence of these small-molecule tyrosine kinase inhibitors (TKIs) provides more possible combinations for neoadjuvant HER2-directed therapy.

Pyrotinib is an irreversible pan-HER TKI targeting HER1, HER2, and HER4. Its anti-tumor activity has been confirmed in patients with previously treated, HER2positive metastatic breast cancer (15-18), and was approved in China in 2018. There is no evidence showing that pyrotinib results in cardiotoxicity. Some phase 2 studies have investigated the application of neoadjuvant pyrotinib in patients with HER2-positive early or locally advanced breast cancer (19-21). Different neoadjuvant combinations were used in these studies to explore the optimal pyrotinibbased regimen. Herein, we investigated the effect of pyrotinib combined with a commonly used chemotherapy regimen (taxane/doxorubicin/cyclophosphamide) as neoadjuvant therapy in patients with HER2-positive locally advanced breast cancer. As the phase 3 GeparSepto-GBG 69 trial demonstrated that substituting the solvent-based paclitaxel with nanoparticle albumin-bound paclitaxel (nabpaclitaxel) followed by epirubicin plus cyclophosphamide before surgery could significantly increase the tpCR rate (29.0\% vs. 38.4\%) in patients with early breast cancer (22), nab-paclitaxel was used in the present study. We present the following article in accordance with the STROBE reporting checklist (available at https://dx.doi. org/10.21037/gs-21-770).

\section{Methods}

\section{Study population}

This was a retrospective case-series study of patients with HER2-positive locally advanced breast cancer who received neoadjuvant therapy with pyrotinib plus chemotherapy at Tangshan People's Hospital between March 2019 and January 2020. The eligibility criteria were as follows: (I) patients aged 18-75 years; (II) those with an Eastern Cooperative Oncology Group (ECOG) performance status $0-1$; (III) treatment-naïve patients; (IV) those with pathologically confirmed HER2-positive (immunohistochemical score of $3+$, or $2+$ with fluorescence in situ hybridization positive) invasive breast cancer; (V) tumor $>2 \mathrm{~cm}$; (VI) patients with a known hormone receptor (HR) status; and (VII) those with complete treatment and follow-up data. This study was performed in accordance with the Declaration of Helsinki (as revised in 2013), and was approved by the Ethics Committee of Tangshan People's Hospital (No. RMYY-LLKS-2021-063). Individual consent for this retrospective analysis was waived.

\section{Treatment}

Poor tolerance to full dose of pyrotinib (400 mg once a day) due to high incidence of diarrhea was observed in clinical practice, leading to discontinuation of pyrotinib and inadequate treatment. Thus, these included patients in our center were treated with pyrotinib $320 \mathrm{mg}$ orally once a day and the TAC regimen (nab-paclitaxel $260 \mathrm{mg} / \mathrm{m}^{2}$, liposomal doxorubicin $20 \mathrm{mg} / \mathrm{m}^{2}$, and cyclophosphamide $600 \mathrm{mg} / \mathrm{m}^{2}$ ) on day 1 of each 21-day cycle. Surgery was performed after 4-6 cycles of neoadjuvant therapy. Adjuvant therapy was decided as per the doctor's discretion. 
Table 1 Baseline characteristics

\begin{tabular}{|c|c|}
\hline Characteristics & Patients $(n=22)$ \\
\hline Age (years), median [range] & 48 [32-60] \\
\hline \multicolumn{2}{|l|}{ Medical history, n (\%) } \\
\hline Hypertension & $1(4.5)$ \\
\hline Diabetes mellitus & $1(4.5)$ \\
\hline Coronary heart disease & $2(9.1)$ \\
\hline \multicolumn{2}{|l|}{ T stage, n (\%) } \\
\hline $\mathrm{T} 2$ & $16(72.7)$ \\
\hline T3 & $6(27.3)$ \\
\hline \multicolumn{2}{|l|}{$\mathrm{N}$ stage, n (\%) } \\
\hline $\mathrm{N} 1$ & $18(81.8)$ \\
\hline N2 & $4(18.2)$ \\
\hline \multicolumn{2}{|l|}{ Clinical stage, n (\%) } \\
\hline IIB & $7(31.9)$ \\
\hline IIIA & $15(68.1)$ \\
\hline \multicolumn{2}{|c|}{ ECOG performance status, $\mathrm{n}(\%)$} \\
\hline 0 & $22(100)$ \\
\hline \multicolumn{2}{|c|}{ Hormone receptor status, n (\%) } \\
\hline ER and/or PgR positive & $10(45.5)$ \\
\hline ER and PgR negative & $12(54.5)$ \\
\hline \multicolumn{2}{|l|}{ Ki-67, n (\%) } \\
\hline$<20 \%$ & 7 (31.8) \\
\hline$\geq 20 \%$ & $15(68.2)$ \\
\hline
\end{tabular}

ECOG, Eastern Cooperative Oncology Group; ER, estrogen receptor; PgR, progesterone receptor.

\section{Data collection}

Demography, disease characteristics, treatment information, and follow-up data were collected from the patients' medical records. Resected tumor samples during surgery were used for pathological response assessment. Outpatient visit or telephone follow-up was censored in December 2020.

\section{Outcomes}

The primary outcome was the tpCR (ypT0/Tis ypN0) rate, defined as the proportion of patients with an absence of invasive cancer components in the breast and without involvement of the axillary lymph nodes. Secondary outcomes included the objective response rate (ORR, defined as the proportion of patients with complete or partial response) after neoadjuvant therapy according to the Response Evaluation Criteria In Solid Tumors version 1.1, progression-free survival (PFS, defined as the time from surgery to disease progression or death from any cause), overall survival (OS, defined as the time from surgery to any-cause death), and the incidence of adverse events (AEs). AEs were graded according to the National Cancer Institute Common Terminology Criteria for Adverse Events, version 5.0 .

\section{Statistical analysis}

Descriptive statistics were used for data analysis. Continuous variables were expressed as the median (range), while categorical variables were expressed as a frequency (percentage). Histograms were used for the analysis of diarrhea.

\section{Results}

A total of 22 patients with complete follow-up data were included for analysis. The median age of the patients was 48 years (range, 32-60). The predominant disease stage was T2 [16 (72.7\%)] and N1 [18 (81.8\%)]. All patients had an ECOG performance status of 0 . Of the 22 patients, 10 $(45.5 \%)$ showed positive estrogen receptor and/or positive progesterone receptor, while $12(54.5 \%)$ exhibited a negative status of both HRs (Table 1).

A total of 16 (72.7\%) patients received four cycles of neoadjuvant therapy, and six $(27.3 \%)$ received six cycles. After the completion of neoadjuvant therapy, 12 (54.5\%) patients achieved a complete response and 10 (45.5\%) achieved a partial response, with an ORR of $100 \%$. The tpCR rate was $45.5 \%(10 / 22)$ after surgery, including four of $10(40.0 \%)$ patients with positive HR and six of 12 (50.0\%) patients with negative HR. By the data cutoff date in December 2020, the median follow-up duration after surgery was 12.5 months (range, 6-20), and no disease recurrence, progression, or death occurred. The 6-month PFS and OS rates were both $100 \%$.

All $22(100 \%)$ patients suffered AEs after neoadjuvant therapy, the majority of which were grade $1-2$. The most common (occurring in $\geq 20 \%$ patients) AEs were diarrhea [21 $(95.5 \%)]$, hand-foot syndrome [21 (95.5\%)], loss of appetite [17 $(77.3 \%)]$, rash [12 (54.5\%)], and asthenia [6 (27.3\%)]. 
Table 2 Adverse events after neoadjuvant therapy

\begin{tabular}{lcc}
\hline \multirow{2}{*}{ Events } & \multicolumn{2}{c}{ Patients $(\mathrm{n}=22)$} \\
\cline { 2 - 3 } & $21(95.5)$ & $4(18.2)$ \\
\hline Diarrhea & $21(95.5)$ & $1(4.5)$ \\
Hand-foot syndrome & $17(77.3)$ & 0 \\
Loss of appetite & $12(54.5)$ & $2(9.1)$ \\
Rash & $6(27.3)$ & 0 \\
Asthenia & $3(13.6)$ & 0 \\
ALT/AST increased & $3(13.6)$ & 0 \\
Platelet count decreased & $3(13.6)$ & 0 \\
Insomnia & $2(9.1)$ & 0 \\
Creatinine increased & $2(9.1)$ & 0 \\
Nausea/vomiting & $2(9.1)$ & 0 \\
Triglycerides increased & $2(9.1)$ & 0 \\
Weight loss & $1(4.5)$ & 0 \\
Cardiotoxicity & &
\end{tabular}

ALT, alanine transaminase; AST, aspartate transaminase.

Grade $\geq 3$ AEs included diarrhea [4 (18.2\%)], rash [2 (9.1\%)], and hand-foot syndrome [1 (4.5\%)] (Table 2). Only one $(4.5 \%)$ patient had a cardiotoxicity event, which was grade 1 .

Of the 21 patients with diarrhea, the first event occurred at 2-6 days after the initiation of neoadjuvant therapy, and $11(52.4 \%)$ patients suffered diarrhea at day 4 (Figure 1A). Grade 3 diarrhea occurred in four patients who recovered to grade 2 or below, and no grade 4 diarrhea was observed (Figure 1B).

\section{Discussion}

This retrospective study investigated the effect of neoadjuvant pyrotinib combined with the TAC regimen (nab-paclitaxel/doxorubicin/cyclophosphamide) in patients with HER2-positive locally advanced breast cancer. The results showed that the ORR before surgery was $100 \%$ and the tpCR rate was $45.5 \%$. This neoadjuvant regimen was also well-tolerated.

Previous randomized controlled trials (NeoALTTO, NeoSphere, and PEONY) showed that mono-targeted therapy with trastuzumab in combination with taxanes provided modest benefit on pathological response, with an tpCR rate of $21.5-27.6 \%(8,13,20)$. Taxanes combined with dual-targeted therapy with trastuzumab plus pertuzumab
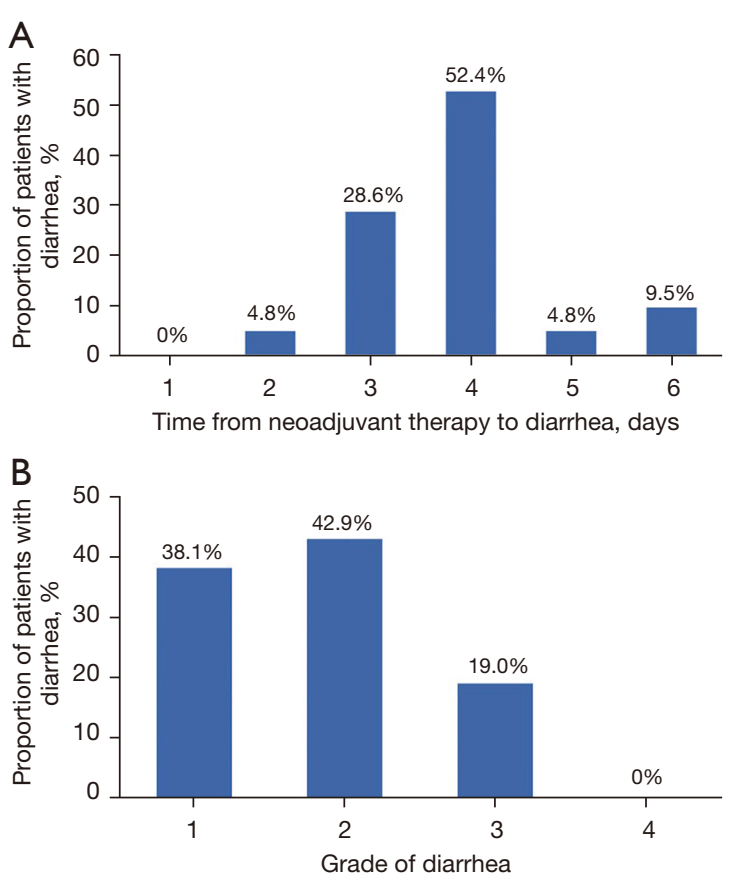

Figure 1 Analysis of diarrhea. (A) Time from the initiation of neoadjuvant therapy to the occurrence of diarrhea. (B) Grade of diarrhea according to the National Cancer Institute Common Terminology Criteria for Adverse Events, version 4.03.

or lapatinib could increase the tpCR rate to $39.3-46.8 \%$ $(8,13,23)$. The randomized phase 3 KRISTINE trial evaluated a new neoadjuvant strategy with antibody-drug conjugate trastuzumab emtansine plus pertuzumab without traditional chemotherapy, and the tpCR rate was $44.4 \%$ (24). The effect of pan-HER TKI pyrotinib combined with chemotherapy on pathological response seemed to be comparable to the aforementioned dual-targeted therapy with or without chemotherapy. However, the tpCR rate in the present study was lower than that with trastuzumab plus pertuzumab and chemotherapy ( $45.5 \%$ vs. $55.7-68.0 \%$ ) in the KRISTINE study and randomized phase 3 TRAIN-2 trial $(24,25)$, which might be due to the addition of carboplatin in both studies and the long treatment cycles (nine cycles) in the TRAIN-2 study. The majority (72.7\%) of patients in the present study only received four cycles of neoadjuvant therapy. Despite the different clinical settings between the studies, the role of pyrotinib as a part of neoadjuvant therapy in HER2-positive breast cancer is worthy of further investigation.

The randomized phase 3 PHOEBE and PHENIX trials have confirmed the significant clinical benefit of pyrotinib 
in HER2-positive metastatic breast cancer $(16,17)$. In the neoadjuvant setting, data from three single-arm clinical trials in patients with HER2-positive early or locally advanced breast cancer have been reported. Liu et al. conducted a two-stage phase 2 trial of neoadjuvant pyrotinib plus docetaxel, carboplatin, and trastuzumab, and the first stage results showed that the tpCR rate was $51.6 \%$ (16/31) (19). Another two phase 2 trials showed that the tpCR rate was $57.1 \%(12 / 21)$ with pyrotinib plus nab-paclitaxel and trastuzumab (20), and $73.7 \%$ (14/19) with pyrotinib plus epirubicin and cyclophosphamide followed by pyrotinib plus docetaxel and trastuzumab (21). Combined with the results of the present study, pyrotinib-based neoadjuvant therapy provides a promising clinical benefit for patients with HER2-positive breast cancer. In addition, it should be noted that the present study was the only one investigating pyrotinib-based neoadjuvant therapy without trastuzumab or pertuzumab. This combination (pyrotinib plus nabpaclitaxel, doxorubicin, and cyclophosphamide) might be an alternative option for patients who could not receive large-molecule monoclonal antibody treatment. Given that the retrospective nature of the present study limited the reliability of results and different combinations were applied among the studies, the efficacy of pyrotinib-based neoadjuvant chemotherapy and the optimal combination need to be verified in large-scale clinical trials.

Small-molecule TKI pyrotinib can be taken orally without concern of cardiotoxicity, but pyrotinib-related diarrhea needs attention. The overall toxicity profile in the present study was acceptable. Diarrhea was the most frequent $\mathrm{AE}$, with an incidence of $95.5 \%$. The first diarrhea event occurred within 6 days after the initiation of neoadjuvant therapy and $52.4 \%$ of patients suffered diarrhea at day 4 . The time to the first episode of diarrhea was early, which was consistent with previous reports in HER2-positive metastatic breast cancer (15-17). The incidence of grade 3 diarrhea in the present study was lower than previously reported results (18.2\% vs. 28.6-64.5\%) from phase 2 studies of pyrotinib-based neoadjuvant therapy (19-21). The dosage at $320 \mathrm{mg}$ rather than $400 \mathrm{mg}$, the use of a single HER2-targeted agent, and no use of carboplatin might have contributed to the low incidence of grade 3 diarrhea. Diarrhea was reversible with antidiarrhea treatment. In addition, since each patient received prophylactic treatment to increase the white blood cell count, no leukopenia occurred in the present study. A mild cardiotoxicity event was observed in one patient.

There were some limitations in this study that should be noted. Firstly, bias could not be avoided due to the retrospective nature and single-center design. Secondly, the sample size was relatively small. Finally, no control group was applied for direct comparison. Nevertheless, we aimed to provide more evidence on pyrotinib-based neoadjuvant therapy in HER2-positive breast cancer for subsequent clinical trials.

In conclusion, neoadjuvant pyrotinib combined with the TAC regimen (nab-paclitaxel/doxorubicin/ cyclophosphamide) showed promising clinical benefit in patients with HER2-positive locally advanced breast cancer, with an acceptable safety profile. Large-molecule monoclonal antibody combined with small-molecule TKI and chemotherapy with different mechanisms of action maybe the mainstay treatment for HER-positive breast cancer in the near future. This combination in our study still warrants further investigation to provide an alternative option for patients who cannot receive large-molecule monoclonal antibody treatment.

\section{Acknowledgments}

Funding: None.

\section{Footnote}

Reporting Checklist: The authors have completed the STROBE reporting checklist. Available at https://dx.doi. org/10.21037/gs-21-770

Data Sharing Statement: Available at https://dx.doi. org/10.21037/gs-21-770

Conflicts of Interest: All authors have completed the ICMJE uniform disclosure form (available at http://dx.doi.org/gs21-770).

Ethical Statement: The authors are accountable for all aspects of the work in ensuring that questions related to the accuracy or integrity of any part of the work are appropriately investigated and resolved. All procedures performed in this study involving human participants were in accordance with the Declaration of Helsinki (as revised in 2013). The study was approved by the Ethics Committee of Tangshan People's Hospital (No. RMYY-LLKS-2021-063). Individual consent for this retrospective analysis was waived.

Open Access Statement: This is an Open Access article 
distributed in accordance with the Creative Commons Attribution-NonCommercial-NoDerivs 4.0 International License (CC BY-NC-ND 4.0), which permits the noncommercial replication and distribution of the article with the strict proviso that no changes or edits are made and the original work is properly cited (including links to both the formal publication through the relevant DOI and the license). See: https://creativecommons.org/licenses/by-nc-nd/4.0/.

\section{References}

1. Sung H, Ferlay J, Siegel RL, et al. Global Cancer Statistics 2020: GLOBOCAN Estimates of Incidence and Mortality Worldwide for 36 Cancers in 185 Countries. CA Cancer J Clin 2021;71:209-49.

2. Zhang S, Sun K, Zheng R, et al. Cancer incidence and mortality in China, 2015. Journal of the National Cancer Center 2021;1:2-11.

3. Cronin KA, Harlan LC, Dodd KW, et al. Populationbased estimate of the prevalence of HER-2 positive breast cancer tumors for early stage patients in the US. Cancer Invest 2010;28:963-8.

4. Gong DH, Ge JY, Chen YY, et al. HER2 overexpression in ductal carcinoma in situ is associated with ipsilateral breast cancer recurrence after conservative surgery. Transl Cancer Res 2020;9:3787-93.

5. Slamon D, Eiermann W, Robert N, et al. Adjuvant trastuzumab in HER2-positive breast cancer. N Engl J Med 2011;365:1273-83.

6. Gianni L, Eiermann W, Semiglazov V, et al. Neoadjuvant and adjuvant trastuzumab in patients with HER2-positive locally advanced breast cancer (NOAH): follow-up of a randomised controlled superiority trial with a parallel HER2-negative cohort. Lancet Oncol 2014;15:640-7.

7. Lv M, Guo H, Wang C, et al. Neoadjuvant docetaxel with or without carboplatin plus dual HER2 blockade for HER2-positive breast cancer: a retrospective multi-center Chinese study. Gland Surg 2020;9:2079-90.

8. Gianni L, Pienkowski T, Im YH, et al. 5-year analysis of neoadjuvant pertuzumab and trastuzumab in patients with locally advanced, inflammatory, or early-stage HER2-positive breast cancer (NeoSphere): a multicentre, open-label, phase 2 randomised trial. Lancet Oncol 2016;17:791-800.

9. Cameron D, Piccart-Gebhart MJ, Gelber RD, et al. 11 years' follow-up of trastuzumab after adjuvant chemotherapy in HER2-positive early breast cancer: final analysis of the HERceptin Adjuvant (HERA) trial. Lancet
2017;389:1195-205.

10. von Minckwitz G, Procter M, de Azambuja E, et al. Adjuvant Pertuzumab and Trastuzumab in Early HER2Positive Breast Cancer. N Engl J Med 2017;377:122-31.

11. National Comprehensive Cancer Network. (NCCN) Clinical Practice Guidelines in Oncology. Breast Cancer, Version 1. 2021. Available online: https://www.nccn.org/ professionals/physician_gls/defaultaspx Accessed 15 Jan 2021.

12. Guidelines of Chinese Society of Clinical Oncology (CSCO). Breast Cancer. 2020. Available online: http:// www.csco.org.cn/cn/index.aspx Accessed 10 Apr 2020.

13. Baselga J, Bradbury I, Eidtmann H, et al. Lapatinib with trastuzumab for HER2-positive early breast cancer (NeoALTTO): a randomised, open-label, multicentre, phase 3 trial. Lancet 2012;379:633-40.

14. Park JW, Liu MC, Yee D, et al. Adaptive Randomization of Neratinib in Early Breast Cancer. N Engl J Med 2016;375:11-22.

15. Ma F, Ouyang Q, Li W, et al. Pyrotinib or Lapatinib Combined With Capecitabine in HER2-Positive Metastatic Breast Cancer With Prior Taxanes, Anthracyclines, and/or Trastuzumab: A Randomized, Phase II Study. J Clin Oncol 2019;37:2610-9.

16. Yan $M$, Bian $L, H u X$, et al. Pyrotinib plus capecitabine for human epidermal factor receptor 2-positive metastatic breast cancer after trastuzumab and taxanes (PHENIX): a randomized, double-blind, placebo-controlled phase 3 study. Transl Breast Cancer Res 2020;1:13.

17. Xu B, Yan M, Ma F, et al. Pyrotinib plus capecitabine versus lapatinib plus capecitabine for the treatment of HER2-positive metastatic breast cancer (PHOEBE): a multicentre, open-label, randomised, controlled, phase 3 trial. Lancet Oncol 2021;22:351-60.

18. Li X, Wu S, Zhang L, et al. HER2-targeted regimens after prior trastuzumab for patients with HER2-positive unresectable, locally advanced or metastatic breast cancer: a network meta-analysis of randomized controlled trials. Ann Transl Med 2020;8:1634.

19. Liu Z, Zhu J, Wang C, et al. 224P Neoadjuvant pyrotinib plus trastuzumab, docetaxel, and carboplatin in patients with HER2-positive early breast cancer: A single-group, multicenter, phase II study. Ann Oncol 2020;31:S331.

20. Luo T, Zhong X, He P, et al. 225P Phase II study of pyrotinib plus albumin-bound paclitaxel and trastuzumab as neoadjuvant treatment in HER2-positive early or locally advanced breast cancer. Ann Oncol 2020;31:S331.

21. Xuhong J, Qi X, Tang P, et al. Neoadjuvant Pyrotinib 
plus Trastuzumab and Chemotherapy for Stage I-III HER2-Positive Breast Cancer: A Phase II Clinical Trial. Oncologist 2020;25:e1909-20.

22. Untch M, Jackisch C, Schneeweiss A, et al. Nabpaclitaxel versus solvent-based paclitaxel in neoadjuvant chemotherapy for early breast cancer (GeparSeptoGBG 69): a randomised, phase 3 trial. Lancet Oncol 2016;17:345-56.

23. Shao Z, Pang D, Yang H, et al. Efficacy, Safety, and Tolerability of Pertuzumab, Trastuzumab, and Docetaxel for Patients With Early or Locally Advanced ERBB2Positive Breast Cancer in Asia: The PEONY Phase 3 Randomized Clinical Trial. JAMA Oncol 2020;6:e193692. 24. Hurvitz SA, Martin M, Symmans WF, et al. Neoadjuvant

Cite this article as: Yao DS, Wang W, Chang JY, Zhang Y, Zhang HW, Xu JX, Cai HF. Neoadjuvant pyrotinib plus nabpaclitaxel, doxorubicin, and cyclophosphamide for HER2positive locally advanced breast cancer: a retrospective caseseries study. Gland Surg 2021;10(12):3362-3368. doi: 10.21037/ gs-21-770 trastuzumab, pertuzumab, and chemotherapy versus trastuzumab emtansine plus pertuzumab in patients with HER2-positive breast cancer (KRISTINE): a randomised, open-label, multicentre, phase 3 trial. Lancet Oncol 2018;19:115-26.

25. van Ramshorst MS, van der Voort A, van Werkhoven ED, et al. Neoadjuvant chemotherapy with or without anthracyclines in the presence of dual HER2 blockade for HER2-positive breast cancer (TRAIN-2): a multicentre, open-label, randomised, phase 3 trial. Lancet Oncol 2018;19:1630-40.

(English Language Editor: A. Kassem) 